\title{
EFEITO DO MÉTODO DE PREPARO DO SOLO, EM ÁREA DE REFORMA, NAS SUAS CARACTERÍSTICAS, NA COMPOSIÇÃO MINERAL E NA PRODUTIVIDADE DE PLANTAÇÕES DE Eucalyptus grandis $^{1}$
}

\author{
Alcides Gatto ${ }^{2}$, Nairam Félix de Barros ${ }^{3}$, Roberto Ferreira de Novais ${ }^{3}$, Liovando Marciano da Costa $^{3} \mathrm{e}$ \\ Júlio César Lima Neves ${ }^{3}$
}

\begin{abstract}
RESUMO - O preparo do solo para o plantio de espécies florestais visa disponibilizar água e nutrientes para o rápido estabelecimento das mudas. Apesar de recentemente técnicas menos intensivas de preparo serem preconizadas, há situações em que o preparo mais intensivo é necessário. Neste trabalho avaliou-se o efeito de quatro métodos de preparo nas propriedades físicas e químicas do solo, na produção de biomassa e na composição mineral de plantação reformada de Eucalyptus grandis. Os tratamentos $\mathrm{T}_{1}$ ) queima dos resíduos da floresta anterior, destoca e subsolagem; $\mathrm{T}_{2}$ ) queima e destoca; $\mathrm{T}_{3}$ ) somente queima; e $\mathrm{T}_{4}$ ) cultivo mínimo (coveamento manual) foram aplicados em área anteriormente usada com plantação de eucalipto manejado por talhadia, em terceira rotação, e situada em Latossolo Vermelho distrófico no município de Santa Bárbara-MG. Aos 38 meses após o plantio, a maior produção de biomassa $(81,6 \mathrm{t} / \mathrm{ha})$ foi verificada no tratamento com maior intensidade de preparo do solo, com decréscimo significativo à medida que o preparo era menos intenso. A menor produtividade $(50,4$ t/ha) foi obtida com o cultivo mínimo. Entretanto, o solo deste tratamento, na época de avaliação, apresentou melhores características químicas e maior acúmulo de manta orgânica. Portanto, o coveamento foi o método de preparo que levaria à maior sustentabilidade da produção florestal, em razão da menor exportação de nutrientes.
\end{abstract}

Palavras-chave: Biomassa, Eucalyptus grandis, preparo do solo e sustentabilidade florestal.

\section{EFECT OF SOIL PREPARATION METHODS ON SOIL CHARACTERISTICS AND MINERAL COMPOSITION AND PRODUCTIVITY OF EUCALYPT}

\begin{abstract}
Tillage system is a silvicultural technique used to increase water and nutrient availability for forest plantation establishment. Low intensity techniques have been recommended in recent years. However, under certain conditions more intensive soil preparation is required. This paper reports the effect of four methods of soil tillage systems on the physical and chemical properties and biomass production and mineral composition of Eucalyptus grandis plantation. The treatments applied were: $T_{1}$ ) slash burning + blade + sub soiling; $T_{2}$ ) slash burning + blade; $T_{3}$ ) slash burning and $T_{4}$ ) unburnt. In all cases, eucalypt seedlings were planted in a hole of $20 \times 20 \times 20 \mathrm{~cm}$. The previous plantation had been conducted by coppicing and the trees harvested three times. The soil was a Red Latosol (Oxisol) located in Santa Barbara, Minas Gerais, Brazil. At the age of 38 months, the largest total biomass $\left(81.6 \mathrm{t} / \mathrm{ha}\right.$ ) was measured in treatment $T_{1}$, decreasing as site preparation intensity was reduced. The lowest yield $(50.4 \mathrm{t} / \mathrm{ha})$ was observed in treatment $T_{4}$. However, the soil under this treatment presented the best chemical characteristics and the largest amount of forest floor, thus favoring forest yield sustainability due to a smaller nutrient removal.
\end{abstract}

Key words: $\quad$ Biomass, Eucalyptus grandis, soil preparation and forest sustainability.

1 Recebido para publicação em 5.8.2002.

Aceito para publicação em 9.9.2003.

Parte da dissertação apresentada pelo primeiro autor para obtenção do grau de mestre em Ciência Florestal, UFV, Viçosa-MG.

2 Doutorando em Solos e Nutrição de Plantas na Universidade Federal de Viçosa - UFV, 36570-000 Viçosa-MG; ${ }^{3}$ Professor do Departamento de Solos - UFV, Bolsista do CNPq. 


\section{INTRODUÇÃO}

O preparo da área e do solo para o plantio de espécies florestais objetiva disponibilizar quantidades suficientes de água e nutrientes para o mais rápido estabelecimento das mudas. Em geral, as técnicas de preparo, além de visar o rápido crescimento do sistema radicular, por meio do revolvimento mais ou menos localizado do solo, o que facilita a absorção de água e de nutrientes, também elimina plantas indesejáveis próximas das mudas da espécie florestal, evitando a competição. É importante, ainda, que a técnica adotada garanta essas condições por período suficientemente longo, para que, mesmo que apareçam, as plantas indesejáveis não venham a competir com a muda.

Até o final da década de 80, o preparo de solo nas áreas reflorestadas consistia na eliminação, em geral por queima, dos resíduos da vegetação anterior e no revolvimento intenso de todo o solo da camada superficial, à semelhança do que se utiliza nos cultivos agrícolas convencionais. Desta forma, o solo ficava desprotegido e sujeito ao processo de erosão, com perdas de solo, e diretamente exposto ao sol (com maior amplitude de temperatura e maior evaporação da água, portanto submetido ao processo de umedecimento e secagem, o que prejudica a estrutura do solo e intensifica a decomposição da matéria orgânica) (Costa, 1990). O crescimento inicial da muda era rápido, o que levava à falsa impressão de excelente e duradoura condição para as plantas. Na realidade, em muitas condições, boa parte dos nutrientes disponibilizados pela queima e pelo revolvimento do solo (decomposição mais rápida da matéria orgânica) era perdida por processos como convecção, erosão e lixiviação. Um trabalho conduzido na região do cerrado mineiro demonstrou que ao final da primeira rotação a perda em volume de madeira em áreas submetidas à queima chegava a mais de $10 \%$ (Maluf, 1991) e que ocorria efeito direto da queima em relação à espécie florestal de interesse, como a degradação do solo pela redução da atividade de biota do solo (Lazari, 2001).

Em meados da década de 80, várias empresas do setor florestal começaram a testar métodos menos intensivos de preparo da área e do solo, por exemplo, sem a queima e com a redução do revolvimento do solo, além da utilização da distribuição dos resíduos do cultivo anterior. Entre as vantagens atribuídas a esse tipo de preparo têm-se a melhoria das características físicas do solo, a redução das perdas de nutrientes, a maior atividade

R. Árvore, Viçosa-MG, v.27, n.5, p.635-646, 2003 biológica e a redução da infestação de plantas invasoras (Gonçalves et al., 2000). Estes autores apresentaram uma revisão detalhada sobre as vantagens e desvantagens do cultivo mínimo em plantações florestais. Lazari (2001) constatou que um povoamento de eucalipto plantado por cultivo mínimo se assemelhava mais à mata nativa quanto à atividade de fosfatases do que um povoamento plantado pelo processo tradicional, que envolvia a queima de resíduos da rotação anterior. Nesse mesmo sentido, Chaer ${ }^{1 /}$ (2001), ao determinar os índices de qualidade do solo em povoamentos de eucalipto submetidos a diferentes intensidades de manejo, comparados ao povoamento de mata natural, encontrou índices de qualidade do solo mais elevados e semelhantes aos da mata para os povoamentos de eucalipto submetidos a manejos menos intensivos.

Apesar das indicações encontradas nesses vários trabalhos sobre a conveniência da adoção de técnicas menos intensivas de preparo da área e do solo, há situações em que o preparo mais intensivo é necessário. Por exemplo, em áreas nas quais várias rotações já foram conduzidas a presença de tocos das antigas árvores tem dificultado a adoção do cultivo mínimo, restringindo ou impossibilitando o trânsito de máquinas e implementos nas várias fases de condução da plantação, em especial na época da colheita, portanto preparos mais intensivos podem ainda ser necessários. Os procedimentos adotados para esse preparo podem variar com as condições locais, mas a queima dos resíduos e a remoção dos tocos têm sido freqüentemente adotadas.

O objetivo deste trabalho foi avaliar o efeito de quatro métodos de preparo do solo, em áreas de reforma de plantações de Eucalyptus grandis W.Hill ex Maiden, sobre as propriedades físicas e químicas do solo, a produção de biomassa e a composição química das árvores.

\section{MATERIAL E MÉTODOS}

\section{1. Área Experimental e Instalação do Experimento}

A área experimental está situada entre as coordenadas geográficas de $19^{\circ} 53^{\prime}$ de latitude sul e $43^{\circ} 21^{\prime}$ de longitude oeste, com altitude aproximada de $735 \mathrm{~m}$, no

1/ CHAER, G. Informação pessoal. Prof. Departamento de Microbiologia Agrícola Universidade Federal de Viçosa. ViçosaMG: UFV (2001). 
município de Santa Bárbara-MG. O clima da região, segundo a classificação de Köppen, é do tipo Cwa, temperado chuvoso-mesotérmico. O solo da área é um Latossolo Vermelho distrófico, textura argilosa, com relevo ondulado.

O experimento foi instalado em uma área de reforma, anteriormente ocupada por um povoamento de eucalipto manejado por talhadia, em terceira rotação. Por isto, os tocos presentes apresentavam grande diâmetro e eram relativamente altos, com cerca de 40 a $50 \mathrm{~cm}$ de altura.

Foram avaliados quatro métodos de preparo do solo:

$$
\begin{aligned}
& \mathrm{T}_{1}=\text { queima }+ \text { destoca }+ \text { subsolagem }(\mathrm{Q}+\mathrm{D}+\mathrm{S}) ; \\
& \mathrm{T}_{2}=\text { queima }+ \text { destoca }(\mathrm{Q}+\mathrm{D}) ; \\
& \mathrm{T}_{3}=\text { queima }(\mathrm{Q}) ; \text { e } \\
& \mathrm{T}_{4}=\text { cultivo mínimo }(\mathrm{CM}) .
\end{aligned}
$$

Nas operações de destoca e subsolagem, foi utilizado um trator de esteira com lâmina KG. A subsolagem foi realizada com subsolador de três hastes, espaçadas $1,0 \mathrm{~m}$ entre si e atingindo a profundidade média de $50-60 \mathrm{~cm}$. A linha de plantio ficou situada no sulco preparado pela haste central. Por razões operacionais, cada tratamento foi aplicado a uma área contínua de aproximadamente 2 ha. Por isto, as avaliações foram realizadas em quatro parcelas amostrais, de $30 \times 20 \mathrm{~m}$ cada $(10 \times 10$ plantas $)$, por tratamento.

No tratamento que envolveu a subsolagem, praticamente não houve necessidade de abertura de cova para o plantio das mudas. Nos demais, foi feita uma cova de aproximadamente $20 \times 20 \times 20 \mathrm{~cm}$, manualmente. $\mathrm{O} \mathrm{T}_{4}$ consistiu apenas na abertura de cova de plantio, com interferência mínima sobre o solo. Os tratos culturais realizados na manutenção da floresta foram os mesmos para todos os tratamentos.

O plantio foi feito em janeiro de 1995, utilizando mudas de Eucalyptus grandis, provenientes de sementes, no espaçamento de $3 \times 2 \mathrm{~m}$. A adubação de plantio consistiu de $100 \mathrm{~g} /$ cova da formulação NPK 5-25$10+0,5 \%$ de boro. Um ano após o plantio, aplicaram-se 100 g/árvore da formulação NPK 10-10-20 sobre a superfície do solo, na projeção da copa, sem incorporação.

O controle de ervas daninhas foi o mesmo para todos os tratamentos, e consistiu de uma aplicação de herbicida pré-emergente (Oxyfluorfen) e uma de pós-emergente (Glyphosate).

\subsection{Avaliações de Campo}

Em cada parcela amostral, procederam-se às avaliações dendrométricas e edáficas. Foram efetuadas medições da circunferência à altura do peito (CAP a $1,30 \mathrm{~m}$ ) de todas as árvores das parcelas, obtendo-se o CAP médio. Em seguida, foi selecionada uma árvore de CAP médio, por parcela, sem falhas na vizinhança, com copa bem formada, que foi abatida para determinação da biomassa. Foram medidos os comprimentos total e comercial do tronco (definidos pelo diâmetro mínimo de $8,0 \mathrm{~cm}$ ). Procedeu-se, também, à cubagem rigorosa do tronco comercial nas posições correspondentes a 0,25 , 50,75 e $100 \%$ do seu comprimento. Em seguida, a árvore foi dividida em lenho, casca, galhos e folhas, sendo estes componentes pesados. Na pesagem dos galhos, foi incluída a ponta da árvore com diâmetro menor que $8,0 \mathrm{~cm}$. Amostras de folhas (200 g), de casca (200 g), obtidas ao longo do tronco, e de galhos (300 g), seccionados em pequenos pedaços, foram coletadas para determinação do peso da matéria seca. Para amostragem do tronco e do lenho, foram coletados serragem e discos de madeira, com casca, de aproximadamente 3,0 cm de espessura, obtidos nas posições correspondentes a 0, 25, 50, 75 e $100 \%$ da altura comercial.

Coletaram-se, ainda, amostras de raízes de eucalipto em cilindros de solo com $22 \mathrm{~cm}$ de diâmetro, nas profundidades de $0-20$ e $20-40 \mathrm{~cm}$. Em cada parcela amostral coletaram-se quatro cilindros de solo, dois na linha de plantio e distanciados 0,5 e $1,0 \mathrm{~m}$ da árvore abatida e dois na entrelinha, distantes 0,5 e 1,5 m da cepa. As raízes, após secagem em estufa, foram classificadas em finas (diâmetro $\leq 3,0 \mathrm{~mm}$ ) e grossas (diâmetro > 3,0 mm). Posteriormente, procedeu-se à medição do comprimento radicular específico (CRE) das raízes finas, usando-se uma placa de vidro com papel milimetrado de quadrículas de $1 \times 1 \mathrm{~cm}(\mathrm{~A})$ colado na face inferior do vidro. O procedimento de medição consistiu em jogar, aleatoriamente, sobre a placa de vidro uma amostra de $1,0 \mathrm{~g}$ de raízes finas, contando-se, posteriormente, o número de intercessões $(\mathrm{N})$ das raízes com as linhas das quadrículas do papel milimetrado. $\mathrm{O}$ comprimento radicular $(\mathrm{C}$, em $\mathrm{cm})$ foi estimado, segundo metodologia proposta por Tennant (1975) e testada por Böhm (1979), usando a equação: C $=11 / 14 \times \mathrm{N} \times \mathrm{A}$.

Amostras de serapilheira (incluindo material de eucalipto e de outras plantas) foram coletadas em dois pontos escolhidos aleatoriamente dentro da parcela,

R. Árvore, Viçosa-MG, v.27, n.5, p.635-646, 2003 
utilizando-se um gabarito com $0,25 \mathrm{~m}^{2}$ de área $(0,5 \times 0,5 \mathrm{~m})$.

As amostras de material vegetal coletadas foram postas para secar em estufa de circulação forçada de ar, à temperatura de $70 \pm 2{ }^{\circ} \mathrm{C}$, até obtenção de peso constante. Com os dados do peso de material fresco e seco das amostras, foi determinada a biomassa dos componentes por árvore e por hectare.

Foram abertas duas trincheiras por parcela, nas dimensões de $60 \times 80 \mathrm{~cm}$, com $60 \mathrm{~cm}$ de profundidade, para coleta de amostras de solo. Amostras de solo deformadas e indeformadas, destinadas às análises químicas e físicas, foram retiradas nas profundidades correspondentes a 0-10, 10-20, 20-30 e 30-40 cm.

Na coleta de amostras indeformadas foram utilizados anéis de PVC rígido, de volume conhecido, com $6,6 \mathrm{~cm}$ de diâmetro interno, $9,5 \mathrm{~cm}$ de altura e $6,0 \mathrm{~mm}$ de espessura.

\subsection{Determinações de Laboratório}

No laboratório, as amostras de tecido vegetal foram submetidas a digestões sulfúrica e nítrico-perclórica. No primeiro estrato foi determinado o teor de $\mathrm{N}$, pelo método de Kjeldahl, e no segundo foram determinados os teores de P, pelo método colorimétrico (Braga \& Defelipo, 1974); de $\mathrm{K}$, por fotometria de chama; e de $\mathrm{Ca}$ e $\mathrm{Mg}$, por espectrofotometria de absorção atômica.

As amostras de solo foram submetidas às seguintes determinações físicas: teor de argila, pelo método da pipeta; densidade do solo (DS), pelo método do anel volumétrico; densidade das partículas (DP), pelo método do balão volumétrico; porosidade total (PT), pelo método indireto; e o equivalente de umidade (EU) (EMBRAPA, 1997). As análises químicas consistiram na determinação de: $\mathrm{pH}$ na relação solo- $\mathrm{H}_{2} \mathrm{O}$ de 1:2,5; fósforo disponível (P), obtido pela extração com Mehlich-1 e determinado por colorimetria; potássio disponível (K), obtido pela extração com Mehlich-1 e determinado por fotometria de emissão de chama; e cálcio, magnésio e alumínio trocáveis, obtidos pela extração com $\mathrm{KCl} 1 \mathrm{~mol} / \mathrm{l}$; na

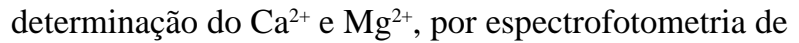
absorção atômica; do $\mathrm{Al}^{3+}$, por titulação; da acidez potencial $(\mathrm{H}+\mathrm{Al})$, pela extração com acetato de cálcio $0,5 \mathrm{~mol} / \mathrm{l}$ a pH 7,0; e na determinação por titulação e carbono orgânico (CO), pelo método de Walkley-Black, seguindo a metodologia descrita por EMBRAPA (1997).

R. Árvore, Viçosa-MG, v.27, n.5, p.635-646, 2003

\subsection{Análises Estatísticas}

$\mathrm{O}$ experimento foi analisado em blocos ao acaso, com quatro repetições. Os dados foram submetidos à análise de variância, utilizando o Sistema para Análises Estatísticas e Genéticas (SAEG). Os graus de liberdade de tratamentos foram desdobrados em três contrastes ortogonais:

$$
\begin{aligned}
& \text { Efeito da queima: } C_{1}=\left(T_{1}+T_{2}+T_{3}\right) \text { vs }\left(T_{4}\right) \text {; } \\
& \text { Efeito da destoca: } C_{2}=\left(T_{1}+T_{2}\right) \text { vs }\left(T_{3}\right) ; e \\
& \text { Efeito da subsolagem: } C_{3}=\left(T_{1}\right) \text { vs }\left(T_{2}\right) .
\end{aligned}
$$

\section{RESULTADOS E DISCUSSÃO}

Os sistemas de preparo adotados, de modo geral, causaram pequenas alterações nas propriedades físicas do solo (Quadro 1). Diferenças significativas entre os contrastes testados foram encontradas principalmente à profundidade de 10-20 cm (Quadro 2). Menores valores de densidade do solo (DS) foram encontrados nas áreas onde a destoca e a subsolagem foram realizadas, como conseqüência do revolvimento do solo promovido por estas práticas de manejo. Em contrapartida, nos tratamentos apenas com queima $\left(\mathrm{T}_{3}\right)$ e cultivo mínimo $\left(\mathrm{T}_{4}\right)$, os valores de DS foram ligeiramente superiores aos das áreas revolvidas. Este fato pode estar relacionado ao antigo sistema de manejo da floresta, por talhadia até a terceira rotação, que não envolveu qualquer tipo de revolvimento do solo, aliado ao tráfego de máquinas durante a colheita da floresta, o que teria favorecido um leve aumento da densidade.

No que tange às características químicas, os valores de $\mathrm{pH}$ foram similares para todos os tratamentos e indicam elevada acidez do solo, em decorrência dos baixíssimos teores de $\mathrm{Ca}$ e $\mathrm{Mg}$ e altos teores de $\mathrm{Al}$ trocável (Quadro 3), características que podem restringir o crescimento das raízes pelas condições desfavoráveis do meio. Apesar de as variações dos valores de $\mathrm{pH}$ serem pequenas, elas tenderam a aumentar com a profundidade para todos os tratamentos. $\mathrm{O}$ aumento dos valores de $\mathrm{pH}$ deve-se à diminuição do teor de matéria orgânica, condição esta geralmente encontrada em solos ácidos (Ardenghi, 1989; Tomé Júnior, 1997).

Os teores de $\mathrm{P}$ e K disponíveis e de Ca e Mg trocáveis decresceram com o aumento da profundidade (Quadro 3), provavelmente em decorrência da ciclagem biogeoquímica, que contribui para aumentar o teor de 
nutrientes na superfície de solos florestais (Binkley, 1986).

Os maiores teores de $\mathrm{P}, \mathrm{K}, \mathrm{Ca}$ e $\mathrm{Mg}$ no solo foram registrados no cultivo mínimo, provavelmente, pela permanência dos restos culturais provenientes da colheita da floresta e pela maior quantidade de serapilheira (do eucalipto e do sub-bosque) presente no local. Ademais, o crescimento das árvores foi menor neste tratamento, fato que leva à menor absorção de nutrientes.

Na região de Santa Bárbara, os resíduos, incluindo a serrapilheira, que permanecem no povoamento após a colheita do tronco somam cerca de $27 \mathrm{t} / \mathrm{ha}$. As quantidades de nutrientes nesses resíduos variam de $10 \mathrm{~kg} / \mathrm{ha}$ de P a $240 \mathrm{~kg} / \mathrm{ha}$ de $\mathrm{N}$ (Leite, 2001), que são liberados

Quadro 1 - Propriedades físicas do solo avaliadas aos 38 meses após o plantio do eucalipto, influenciadas pelo preparo do solo utilizado na reforma

Table 1 - Soil physical properties, evaluated 38 months after eucalypt planting, as affected by soil tillage systems

\begin{tabular}{|c|c|c|c|c|c|}
\hline \multirow{2}{*}{ Trat. } & Prof. & DS & DP & PT & EU \\
\hline & $(\mathrm{cm})$ & \multicolumn{2}{|c|}{$\left(\mathrm{g} / \mathrm{cm}^{3}\right)$} & \multicolumn{2}{|c|}{$(\%)$} \\
\hline \multirow{3}{*}{$\mathrm{T}_{1}$} & $0-10$ & $\begin{array}{c}0,98 \\
( \pm 0,03)\end{array}$ & $\begin{array}{c}2,62 \\
( \pm 0,09)\end{array}$ & $\begin{array}{c}63 \\
( \pm 1,5)\end{array}$ & $\begin{array}{c}25,2 \\
( \pm 2,4)\end{array}$ \\
\hline & $10-20$ & $\begin{array}{c}1,07 \\
( \pm 0,04)\end{array}$ & $\begin{array}{c}2,45 \\
( \pm 0,08)\end{array}$ & $\begin{array}{c}56 \\
( \pm 1,2)\end{array}$ & $\begin{array}{c}26,1 \\
( \pm 3,2)\end{array}$ \\
\hline & $20-40$ & $\begin{array}{c}1,09 \\
( \pm 0,05)\end{array}$ & $\begin{array}{c}2,56 \\
( \pm 0,10)\end{array}$ & $\begin{array}{c}57 \\
( \pm 0,8)\end{array}$ & $\begin{array}{c}26,8 \\
( \pm 2,6)\end{array}$ \\
\hline \multirow{3}{*}{$\mathrm{T}_{2}$} & $0-10$ & $\begin{array}{c}0,91 \\
( \pm 0,03) \\
\end{array}$ & $\begin{array}{c}2,50 \\
( \pm 0,03)\end{array}$ & $\begin{array}{c}64 \\
( \pm 1,5)\end{array}$ & $\begin{array}{c}25,3 \\
( \pm 1,5)\end{array}$ \\
\hline & $10-20$ & $\begin{array}{c}0,95 \\
( \pm 0,02) \\
\end{array}$ & $\begin{array}{c}2,41 \\
( \pm 0,05) \\
\end{array}$ & $\begin{array}{c}60 \\
( \pm 0,7) \\
\end{array}$ & $\begin{array}{c}25,8 \\
( \pm 1,3) \\
\end{array}$ \\
\hline & $20-40$ & $\begin{array}{c}0,94 \\
( \pm 0,03)\end{array}$ & $\begin{array}{c}2,56 \\
( \pm 0,04)\end{array}$ & $\begin{array}{c}63 \\
( \pm 1,6)\end{array}$ & $\begin{array}{c}25,9 \\
( \pm 1,6)\end{array}$ \\
\hline \multirow{3}{*}{$\mathrm{T}_{3}$} & $0-10$ & $\begin{array}{c}1,01 \\
( \pm 0,05) \\
\end{array}$ & $\begin{array}{c}2,42 \\
( \pm 0,02)\end{array}$ & $\begin{array}{c}58 \\
( \pm 1,6)\end{array}$ & $\begin{array}{c}28,9 \\
( \pm 0,8)\end{array}$ \\
\hline & $10-20$ & $\begin{array}{c}1,04 \\
( \pm 0,02)\end{array}$ & $\begin{array}{c}2,47 \\
( \pm 0,07)\end{array}$ & $\begin{array}{c}58 \\
( \pm 0,6)\end{array}$ & $\begin{array}{c}29,7 \\
( \pm 0,3)\end{array}$ \\
\hline & $20-40$ & $\begin{array}{c}1,05 \\
( \pm 0,04) \\
\end{array}$ & $\begin{array}{c}2,56 \\
( \pm 0,03) \\
\end{array}$ & $\begin{array}{c}59 \\
( \pm 1,2) \\
\end{array}$ & $\begin{array}{c}30,3 \\
( \pm 0,6) \\
\end{array}$ \\
\hline \multirow{3}{*}{$\mathrm{T}_{4}$} & $0-10$ & $\begin{array}{c}1,03 \\
( \pm 0,03) \\
\end{array}$ & $\begin{array}{c}2,70 \\
( \pm 0,06)\end{array}$ & $\begin{array}{c}62 \\
( \pm 1,3)\end{array}$ & $\begin{array}{c}25,8 \\
( \pm 0,4)\end{array}$ \\
\hline & $10-20$ & $\begin{array}{c}1,09 \\
( \pm 0,01)\end{array}$ & $\begin{array}{c}2,73 \\
( \pm 0,02) \\
\end{array}$ & $\begin{array}{c}60 \\
( \pm 0,6)\end{array}$ & $\begin{array}{c}25,5 \\
( \pm 0,3)\end{array}$ \\
\hline & $20-40$ & $\begin{array}{c}1,07 \\
( \pm 0,01)\end{array}$ & $\begin{array}{c}2,76 \\
( \pm 0,08)\end{array}$ & $\begin{array}{c}61 \\
( \pm 1,5)\end{array}$ & $\begin{array}{c}26,1 \\
( \pm 0,3)\end{array}$ \\
\hline
\end{tabular}

DS = densidade do solo; $\mathrm{DP}=$ densidade das partículas, $\mathrm{PT}=$ porosidade total e $\mathrm{EU}=$ equivalente umidade. Os valores entre parêntesis equivalem ao erro-padrão da média. para o sistema de forma lenta e gradual, por meio da mineralização. Portanto, qualquer técnica de manejo do solo que venha acelerar o processo de mineralização, como a queima e o revolvimento do solo, a exemplo dos tratamentos $\mathrm{T}_{1}, \mathrm{~T}_{2} \mathrm{e} \mathrm{T}_{3}$, contribui para a disponibilização mais rápida de nutrientes. Tal fato pode ter reflexo na produtividade do povoamento, dependendo se os nutrientes são absorvidos pelas arvores ou, de alguma forma, perdidos do ecossistema.

Vale ressaltar que, independentemente do tratamento aplicado, os teores de todos os nutrientes no solo são baixos, aquém daqueles considerados adequados, mesmo para implantação de florestas de eucalipto, à exceção do K (Barros et al., 1990). A fertilidade do solo seria insuficiente para suportar produtividade superior a $10 \mathrm{~m}^{3} / \mathrm{ha} /$ ano na próxima rotação (Novais et al., 1986). Por exemplo, o conteúdo de Ca na biomassa reportado por Leite (2001), para a região deste estudo, foi de $204 \mathrm{~kg} / \mathrm{ha}$, para um povoamento de eucalipto com idade próxima de 7 anos de idade, ao passo que em regiões mais produtivas essa quantidade atingiria mais de 400 kg/ha (Santana \& Neves, 1999; Neves, 2001), o que reforça a baixa disponibilidade desse nutriente. Deste modo, mais do que corrigir a acidez do solo, reduzir e, ou, neutralizar os efeitos nocivos do alumínio, a calagem pode ser necessária para elevar a disponibilidade de $\mathrm{Ca}$ e de Mg.

As características dendrométricas, entre as quais estão a sobrevivência e o volume do tronco, foram afetadas de forma significativa pelos sistemas de preparo do solo utilizados para a reforma (Quadros 4 e 5). De modo geral, houve tendência de aumento da sobrevivência, da circunferência e da altura da árvore, com reflexo no volume, à medida que se intensificou o preparo do solo. Maluf (1991) também constatou, para tratamentos similares, maior produtividade da floresta à medida que os sistemas de preparo do solo se intensificavam. Ele obteve, aos 51 meses após o plantio, superioridade de $77 \%$ quando o preparo foi realizado com dupla gradagem com grade pesada, em relação ao preparo com grade bedding. Essa diferença em crescimento foi atribuída à maior infestação de ervas daninhas no preparo menos intensivo.

O maior crescimento observado nos tratamentos de preparo mais intensivo do solo pode ser atribuído às melhores condições criadas para absorção de nutrientes e de água pelas plantas de eucalipto e pela redução da competição com ervas daninhas.

R. Árvore, Viçosa-MG, v.27, n.5, p.635-646, 2003 
Quadro 2 - Análise da variância para as características físicas do solo, influenciadas pelo preparo do solo utilizado na reforma da floresta

Table 2 - Analysis of variance for soil physical characteristics, according to soil tillage systems

\begin{tabular}{|c|c|c|c|c|c|}
\hline \multirow{2}{*}{$\mathrm{FV}$} & \multirow{2}{*}{ GL } & \multicolumn{4}{|c|}{ Quadrado Médio } \\
\hline & & DS & $\mathrm{DP}$ & $\mathrm{PT}$ & EU \\
\hline & & \multicolumn{4}{|c|}{ Prof. $0-10 \mathrm{~cm}$} \\
\hline$\left(T_{1}, T_{2}\right.$ e $\left.T_{3}\right)$ vs $\left(T_{4}\right)$ & 1 & $0,01 \mathrm{~ns}$ & $0,10^{*}$ & $0,08 \mathrm{~ns}$ & $1,23 \mathrm{~ns}$ \\
\hline$\left(T_{1}\right.$ e $\left.T_{2}\right)$ vs $\left(T_{3}\right)$ & 1 & $0,01 \mathrm{~ns}$ & $0,05 \mathrm{~ns}$ & $68,92 *$ & $35,25 \mathrm{~ns}$ \\
\hline$\left(T_{1}\right)$ vs $\left(T_{2}\right)$ & 1 & $0,01 \mathrm{~ns}$ & $0,03 \mathrm{~ns}$ & $2,28 \mathrm{~ns}$ & $0,03 \mathrm{~ns}$ \\
\hline $\begin{array}{r}\text { Resíduo } \\
\end{array}$ & & 0,01 & 0,02 & 10,79 & 8,02 \\
\hline \multirow[t]{2}{*}{$\mathrm{CV}(\%)$} & & 8,45 & 4,95 & 5,34 & 10,77 \\
\hline & & \multicolumn{4}{|c|}{ Prof. $10-20 \mathrm{~cm}$} \\
\hline$\left(T_{1}, T_{2}\right.$ e $\left.T_{3}\right)$ vs $\left(T_{4}\right)$ & 1 & $0,01 *$ & $0,25 * *$ & $9,19 \mathrm{~ns}$ & $8,75 \mathrm{~ns}$ \\
\hline$\left(\mathrm{T}_{1} \mathrm{e} \mathrm{T}_{2}\right) \mathrm{vs}\left(\mathrm{T}_{3}\right)$ & 1 & $0,00 \mathrm{~ns}$ & $0,01 \mathrm{~ns}$ & $0,80 \mathrm{~ns}$ & $38,39 \mathrm{~ns}$ \\
\hline$\left(T_{1}\right)$ vs $\left(T_{2}\right)$ & 1 & $0,03 * *$ & $0,00 \mathrm{~ns}$ & $32,68 * *$ & $0,19 \mathrm{~ns}$ \\
\hline Resíduo & & 0,00 & 0,01 & 2,87 & 11,47 \\
\hline \multirow[t]{2}{*}{$\mathrm{CV}(\%)$} & & 4,76 & 4,68 & 2,89 & 12,66 \\
\hline & & \multicolumn{4}{|c|}{ Prof. $20-40 \mathrm{~cm}$} \\
\hline$\left(T_{1}, T_{2}\right.$ e $\left.T_{3}\right)$ vs $\left(T_{4}\right)$ & 1 & $0,01 \mathrm{~ns}$ & $0,12 *$ & $4,15 \mathrm{~ns}$ & $7,29 \mathrm{~ns}$ \\
\hline$\left(\mathrm{T}_{1} \mathrm{e} \mathrm{T}_{2}\right) \mathrm{vs}\left(\mathrm{T}_{3}\right)$ & 1 & $0,00 \mathrm{~ns}$ & $0,00 \mathrm{~ns}$ & $4,64 \mathrm{~ns}$ & $41,30 \mathrm{~ns}$ \\
\hline$\left(T_{1}\right)$ vs $\left(T_{2}\right)$ & 1 & $0,04 \mathrm{~ns}$ & $0,00 \mathrm{~ns}$ & $64,19 \mathrm{~ns}$ & $1,73 \mathrm{~ns}$ \\
\hline Resíduo & & 0,02 & 0,02 & 8,63 & 11,16 \\
\hline $\mathrm{CV}(\%)$ & & 6,55 & 4,95 & 4,89 & 12,25 \\
\hline
\end{tabular}

$\mathrm{FV}=$ fonte de variação, $\mathrm{GL}=$ graus de liberdade, $\mathrm{DS}=$ densidade do solo, $\mathrm{DP}=$ densidade das partículas, $\mathrm{PT}=$ porosidade total, $\mathrm{EU}=$ equivalente umidade, $\mathrm{CV}=$ coeficiente de variação; e ns, * e ** = não-significativo e significativos a 5 e $1 \%$, respectivamente.

Quadro 3 - Características químicas do solo avaliadas aos 38 meses após o plantio do eucalipto, influenciadas pelo preparo do solo utilizado na reforma da floresta

Table 3 - Soil chemical characteristics evaluated 38 months after eucalypt planting, as affected by soil tillage systems

\begin{tabular}{|c|c|c|c|c|c|c|c|c|c|c|c|c|c|c|}
\hline \multirow{2}{*}{ Trat. } & Prof. & \multirow{2}{*}{$\mathrm{pH}$} & $\mathrm{P}$ & $\mathrm{K}$ & $\mathrm{Ca}^{2+}$ & $\mathrm{Mg}^{2+}$ & $\mathrm{Al}^{3+}$ & $\mathrm{H}+\mathrm{Al}$ & SB & $\mathrm{t}$ & $\mathrm{T}$ & $\mathrm{V}$ & $\mathrm{m}$ & \multirow{2}{*}{$\frac{\mathrm{CO}}{(\mathrm{dag} / \mathrm{kg})}$} \\
\hline & $(\mathrm{cm})$ & & \multicolumn{2}{|c|}{$\left(\mathrm{mg} / \mathrm{dm}^{3}\right)$} & \multicolumn{7}{|c|}{$\left(\mathrm{cmol}_{\mathrm{C}} / \mathrm{dm}^{3}\right)$} & \multicolumn{2}{|c|}{$(\%)$} & \\
\hline \multirow{3}{*}{$\mathrm{T}_{1}$} & $0-10$ & 4,3 & 0,87 & 20,8 & 0,041 & 0,07 & 2,1 & 9,6 & 0,3 & 2,3 & 9,7 & 1,8 & 92,4 & 3,0 \\
\hline & $10-20$ & 4,4 & 0,65 & 15,5 & 0,015 & 0,06 & 1,7 & 8,7 & 0,1 & 1,9 & 8,8 & 1,3 & 94,0 & 2,1 \\
\hline & $20-40$ & 4,5 & 0,46 & 13,3 & 0,007 & 0,04 & 1,5 & 7,0 & 0,1 & 1,5 & 7,1 & 1,2 & 94,7 & 1,4 \\
\hline \multirow{3}{*}{$\mathrm{T}_{2}$} & $0-10$ & 4,2 & 0,68 & 17,8 & 0,008 & 0,05 & 1,8 & 9,6 & 0,1 & 1,9 & 9,7 & 1,1 & 94,4 & 3,4 \\
\hline & $10-20$ & 4,2 & 0,76 & 19,0 & 0,007 & 0,05 & 1,7 & 9,1 & 0,1 & 1,8 & 9,3 & 1,1 & 94,2 & 3,7 \\
\hline & $20-40$ & 4,3 & 0,50 & 11,3 & 0,003 & 0,03 & 1,1 & 9,7 & 0,1 & 1,2 & 9,8 & 0,7 & 94,7 & 2,1 \\
\hline \multirow{3}{*}{$\mathrm{T}_{3}$} & $0-10$ & 4,2 & 0,98 & 24,5 & 0,056 & 0,08 & 2,5 & 8,3 & 0,2 & 2,8 & 8,5 & 2,4 & 92,6 & 3,5 \\
\hline & $10-20$ & 4,3 & 0,57 & 13,5 & 0,010 & 0,05 & 2,1 & 6,7 & 0,1 & 2,2 & 6,8 & 1,4 & 95,5 & 2,4 \\
\hline & $20-40$ & 4,4 & 0,46 & 8,0 & 0,007 & 0,04 & 1,7 & 9,8 & 0,1 & 1,7 & 9,9 & 0,7 & 96,4 & 1,7 \\
\hline \multirow{3}{*}{$\mathrm{T}_{4}$} & $0-10$ & 4,2 & 1,02 & 28,0 & 0,085 & 0,10 & 1,8 & 10,7 & 0,3 & 2,0 & 11,0 & 2,3 & 87,5 & 3,1 \\
\hline & $10-20$ & 4,3 & 0,54 & 16,0 & 0,035 & 0,06 & 1,3 & 8,9 & 0,1 & 1,4 & 9,1 & 1,5 & 89,8 & 2,2 \\
\hline & $20-40$ & 4,4 & 0,46 & 11,8 & 0,011 & 0,04 & 1,0 & 7,8 & 0,1 & 1,1 & 7,9 & 1,2 & 91,1 & 1,7 \\
\hline
\end{tabular}

$\mathrm{pH}$ - relação solo:água 1:2,5; P e K - extrator Mehlich-1; $\mathrm{Ca}^{2+}, \mathrm{Mg}^{2+}$ e $\mathrm{Al}^{3+}$ - extrator $\mathrm{KCl} 1 \mathrm{~mol} / \mathrm{l} ; \mathrm{H}+\mathrm{Al}$ - extrator acetato de cálcio $0,5 \mathrm{~mol} / \mathrm{l}$ a $\mathrm{pH} 7,0 ; \mathrm{SB}=$ soma de bases; $\mathrm{t}=$ capacidade de troca catiônica efetiva; $\mathrm{T}=$ capacidade de troca catiônica total a pH 7,0; V = índice de saturação por bases; $\mathrm{m}$ = índice de saturação por alumínio; e CO = carbono orgânico. 
O estabelecimento inicial das mudas e seu crescimento pós-plantio são favorecidos pela queima dos resíduos e pelo revolvimento do solo, que causam aumento na disponibilidade de nutrientes, com conseqüente maior crescimento das raízes e de área foliar para captura de nutrientes e energia solar, respectivamente.

A capacidade produtiva de uma floresta está intimamente relacionada com o crescimento horizontal e vertical de raízes no solo e é dependente do potencial genético da espécie, do clima, das características do solo e dos tratos culturais utilizados para sua implantação e manutenção (Reis \& Reis, 1995). O crescimento radicular específico (CRE) indica a superfície de absorção de água e nutrientes por parte das raízes finas (diâmetro $\leq 3,0 \mathrm{~mm}$ ). Os tratamentos que resultaram em maiores produtividades de biomassa foram aqueles que apresentaram maior CRE, reflexo direto da maior área de absorção de água e nutrientes pelas plantas. O CRE médio do eucalipto obtido para os quatro sistemas de manejo foi de $488,0 \mathrm{~cm} / \mathrm{g}$ de raiz seca. Os tratamentos que envolvem maior intensidade de preparo permitiram maior CRE, ao passo que os de menor intensidade resultaram em menor CRE. No $\mathrm{T}_{1}$ o CRE médio estimado foi de $516,4 \mathrm{~cm} / \mathrm{g}$, no $\mathrm{T}_{2}$ de $494,8 \mathrm{~cm} / \mathrm{g}$ e no $\mathrm{T}_{3}$ de $476,9 \mathrm{~cm} / \mathrm{g}$, e o menor foi no $\mathrm{T}_{4} \mathrm{com} 463,3 \mathrm{~cm} / \mathrm{g}$ de raiz seca. O menor CRE do eucalipto no $\mathrm{T}_{4}$ justifica-se pela competição exercida pelas ervas daninhas por água e nutrientes, além dos maiores valores de resistência à penetração das raízes verificados, o que provavelmente teria dificultado a expansão do sistema radicular, com reflexos diretos sobre a produção de biomassa.

Nos tratamentos onde foram realizadas a destoca e a subsolagem houve incorporação dos resíduos culturais não-queimados do cultivo anterior e das cinzas provenientes da queima de parte desses resíduos. Desta forma, as perdas de nutrientes pelo escoamento superficial das águas de chuva e pelo transporte de partículas pelo vento foram reduzidas, refletindo-se positivamente na produção de biomassa pela maior oferta de nutrientes. A infestação por ervas daninhas, em geral, também é menor quando se prepara o solo, o que contribui para o maior crescimento inicial das árvores, em razão da redução da competição das mudas com as ervas daninhas por água e nutrientes, principalmente nos estádios iniciais de crescimento. Segundo Scheneider (1993), a competição entre as plantas de interesse com as ervas daninhas por luz, água e nutrientes pode ser considerada como o fator biológico mais importante no manejo da floresta.
O menor crescimento verificado no cultivo mínimo $\left(\mathrm{T}_{4}\right)$ pode ser atribuído à maior resistência à penetração de raízes e à menor disponibilidade de nutrientes para as mudas. Como grande parte dos nutrientes presentes no sistema está no material orgânico depositado sobre o solo, com decomposição mais lenta e gradual que os demais sistemas, pela não-queima ou incorporação do material vegetal, os organismos decompositores competem pelos nutrientes disponíveis com as mudas, reduzindo seu vigor e crescimento (Gonçalves, 1995; Gonçalves et al., 2000). Segundo Gonçalves (1995), com o passar do tempo, o vigor e a homogeneidade de crescimento da floresta, quando se adota o cultivo mínimo, tendem a se restabelecer, dependendo, porém, da qualidade do sítio. Em sítios de melhor qualidade, geralmente as plantas atingem crescimento equivalente ao observado em sistemas de preparo mais intensivo do solo, por volta de 12 a 24 meses pós-plantio.

A produção de biomassa de árvores de $E$. grandis aos 38 meses de idade foi significativamente alterada pelo tipo de preparo do solo utilizado para a reforma da floresta (Quadros 4 e 5). De modo geral, a produção de biomassa decresceu com a redução da intensidade de preparo por ocasião da reforma. $\mathrm{O} \mathrm{T}_{1}$ permitiu maior produção de biomassa para todos os componentes da parte aérea e total, seguido dos tratamentos $\mathrm{T}_{2} \mathrm{e} \mathrm{T}_{3}$. A maior produção nesses tratamentos pode estar ligada à melhoria das propriedades físicas, o que permitiria maior expansão do sistema radicular para maiores profundidades, além de maior CRE, conforme já discutido.

As árvores tenderam a alocar maior proporção de biomassa na copa à medida que o preparo do solo foi menos intensivo (Quadro 4). Este fato tem sido comumente observado quando é maior a restrição na disponibilidade de água e nutrientes e, conseqüentemente, maior proporção de fotoassimilados é destinada ao sistema radicular (Leite, 2001). Neste experimento, nos tratamentos referentes à queima e ao cultivo mínimo houve tendência de aumento de biomassa de raízes em relação ao preparo mais intensivo, embora a diferença não tenha sido significativa (Quadro 5). Além disto, em razão de o crescimento em diâmetro ser menor, a altura comercial foi menor nas árvores destes tratamentos, o que resultou em maior quantidade de copa.

A maior quantidade de manta orgânica registrada no $\mathrm{T}_{4}$ (Quadro 4) deve-se à não-queima dos resíduos culturais e à sua permanência na superfície do solo, o que reduz sua mineralização pela menor superfície de

R. Árvore, Viçosa-MG, v.27, n.5, p.635-646, 2003 
ataque dos microrganismos. A manta orgânica (manta orgânica + sub-bosque) libera os nutrientes de forma lenta e gradual, tendo em vista sua relação $\mathrm{C} / \mathrm{N}$, em torno de 56 , que favorece mais a imobilização do que a mineralização de nutrientes.

Pelos contrastes ortogonais testados (Quadro 5), observaram-se diferenças significativas na produção de biomassa dos componentes da planta em decorrência dos sistemas de preparo do solo utilizados. A queima dos resíduos da plantação anterior e o preparo do solo concorreram para o aumento da produtividade $\left(\mathrm{T}_{1}+\mathrm{T}_{2} \mathrm{~T}_{3}\right.$ vs $\left.\mathrm{T}_{4}\right)$, já a queima combinada com a destoca não afetou significativamente a produção, quando comparada com a queima. Contudo, esta técnica elevou a produção total de biomassa, em média $11,6 \%$ em relação ao cultivo mínimo. A subsolagem ( $\mathrm{T}_{1}$ vs $\mathrm{T}_{2}$ ) contribuiu de modo significativo para o aumento da produtividade, 27,1 e $21,7 \%$ na biomassa de tronco e total, respectivamente.

À idade de avaliação deste experimento, o $\mathrm{N}$ foi o nutriente que mais se acumulou na árvore, independentemente do tipo de preparo, seguido por $\mathrm{Ca}, \mathrm{K}, \mathrm{Mg}$ e $\mathrm{P}$ (Quadro 6). A mesma seqüência de concentração, tanto para as espécies florestais nativas como para o eucalipto, foi obtida por Golley et al. (1978) e Drumomd (1996). O conteúdo de $\mathrm{K}$ foi significativamente afetado pelo tipo de preparo do solo, sendo ele crescente nos preparos mais intensivos.

Quadro 4 - Sobrevivência, volume de tronco e biomassa dos componentes de árvores de Eucalyptus grandis, aos 38 meses de idade, influenciados pelo preparo do solo utilizado para a reforma da floresta

Table 4 - Survival, stem volume and biomass in the components of 38-month-old Eucalyptus grandis, as affected by soil tillage systems

\begin{tabular}{|c|c|c|c|c|c|c|c|}
\hline \multirow{2}{*}{ Trat. } & Sobrevivência & Tronco & Copa & Tronco & MO & $\mathrm{Rg}$ & $\mathrm{Rf}$ \\
\hline & $(\%)$ & $\left(\mathrm{m}^{3} / \mathrm{ha}\right)$ & \multicolumn{5}{|c|}{ (t/ha) } \\
\hline $\mathrm{T}_{1}$ & $\begin{array}{c}88,5 \\
( \pm 2,5)^{1^{\prime}}\end{array}$ & $\begin{array}{c}135,18 \\
( \pm 4,87)\end{array}$ & $\begin{array}{c}9,07 \\
( \pm 0,39)\end{array}$ & $\begin{array}{c}55,57 \\
( \pm 3,15)\end{array}$ & $\begin{array}{c}13,67 \\
( \pm 0,65)\end{array}$ & $\begin{array}{c}2,86 \\
( \pm 0,50)\end{array}$ & $\begin{array}{c}0,41 \\
( \pm 0,03)\end{array}$ \\
\hline $\mathrm{T}_{2}$ & $\begin{array}{c}81,0 \\
( \pm 3,7)\end{array}$ & $\begin{array}{c}101,50 \\
( \pm 10,58)\end{array}$ & $\begin{array}{c}7,84 \\
( \pm 0,42)\end{array}$ & $\begin{array}{c}40,51 \\
( \pm 4,19)\end{array}$ & $\begin{array}{c}12,92 \\
( \pm 0,56)\end{array}$ & $\begin{array}{c}1,98 \\
( \pm 0,09)\end{array}$ & $\begin{array}{c}0,60 \\
( \pm 0,10)\end{array}$ \\
\hline $\mathrm{T}_{3}$ & $\begin{array}{c}80,5 \\
( \pm 0,8) \\
\end{array}$ & $\begin{array}{c}89,98 \\
( \pm 4,14)\end{array}$ & $\begin{array}{c}8,31 \\
( \pm 0,28)\end{array}$ & $\begin{array}{c}37,11 \\
( \pm 1,92)\end{array}$ & $\begin{array}{c}10,49 \\
( \pm 0,39)\end{array}$ & $\begin{array}{c}6,08 \\
( \pm 2,75)\end{array}$ & $\begin{array}{c}0,51 \\
( \pm 0,08)\end{array}$ \\
\hline $\mathrm{T}_{4}$ & $\begin{array}{c}71,2 \\
( \pm 2,5)\end{array}$ & $\begin{array}{c}63,43 \\
135,18\end{array}$ & $\begin{array}{c}7,65 \\
( \pm 0,35)\end{array}$ & $\begin{array}{c}24,89 \\
( \pm 3,03)\end{array}$ & $\begin{array}{c}14,75 \\
( \pm 0,64)\end{array}$ & $\begin{array}{c}2,69 \\
( \pm 0,66)\end{array}$ & $\begin{array}{l}0,40 \\
0,41\end{array}$ \\
\hline
\end{tabular}

Tronco $=($ casca + lenho $)$, Copa $=($ folha + galho $), \mathrm{MO}=$ manta orgânica, $\mathrm{Rg}=$ raízes grossas $($ diâmetro $>3,00 \mathrm{~mm})$ e $\mathrm{Rf}=$ raízes finas (diâmetro $\leq 3,00 \mathrm{~mm}) \cdot{ }^{1 /}=$ erro-padrão da média.

Quadro 5 - Análise de variância da sobrevivência, do volume do tronco e da produção de biomassa dos componentes de plantas de Eucalyptus grandis, aos 38 meses de idade, influenciados pelo preparo do solo utilizado para a reforma da floresta

Table 5 - Analysis of variance of Eucalyptus grandis survival, stem volume and biomass in the components of eucalyptus trees, at 38 months of age, as affected by soil tillage systems

\begin{tabular}{|c|c|c|c|c|c|c|c|c|}
\hline \multirow{2}{*}{ FV } & \multirow{2}{*}{ GL } & \multicolumn{7}{|c|}{ Quadrado Médio } \\
\cline { 3 - 9 } & & Sobrevivência & Tronco & Copa & Tronco & PA & MO & Raízes \\
\hline$\left(\mathrm{T}_{1}, \mathrm{~T}_{2} \mathrm{e} \mathrm{T}_{3}\right)$ vs $\left(\mathrm{T}_{4}\right)$ & 1 & $437,85^{* *}$ & $6.199,94^{* *}$ & $0,66 \mathrm{~ns}$ & $1.141,50^{* *}$ & $1.232,30^{* *}$ & $17,16^{*}$ & $3,31 \mathrm{~ns}$ \\
\hline$\left(\mathrm{T}_{1} \mathrm{e} \mathrm{T}_{2}\right)$ vs $\left(\mathrm{T}_{3}\right)$ & 1 & $48,15 \mathrm{~ns}$ & $2.145,09^{*}$ & $0,03 \mathrm{~ns}$ & $318,66^{*}$ & $327,36^{*}$ & $20,91^{*}$ & $35,60 \mathrm{~ns}$ \\
\hline$\left(\mathrm{T}_{1}\right)$ vs $\left(\mathrm{T}_{2}\right)$ & 1 & $112,46 \mathrm{~ns}$ & $2.267,71^{*}$ & $0,75 \mathrm{~ns}$ & $453,27^{*}$ & $530,16^{*}$ & $1,12 \mathrm{~ns}$ & $0,94 \mathrm{~ns}$ \\
\hline Resíduo & & 29,72 & 259,13 & 0,60 & 45,39 & 62,66 & 1,67 & 9,47 \\
\hline CV $(\%)$ & & 6,79 & 16,51 & 19,89 & 17,05 & 16,58 & 9,97 & 79,26 \\
\hline
\end{tabular}

$\mathrm{FV}=$ fonte de variação, $\mathrm{GL}=$ graus de liberdade, $\mathrm{CV}=$ coeficiente de variação; e ns, * e ** = não-significativo e significativos a 5 e $1 \%$, respectivamente. 
Quadro 6 - Conteúdo de nutrientes nos componentes de árvores de Eucalyptus grandis, aos 38 meses de idade, influenciado pelo preparo do solo adotado para reforma de floresta

Table 6 - Amount of nutrients in the components of Eucalyptus grandis trees at 38 months of age, as affected by soil tillage systems

\begin{tabular}{|c|c|c|c|c|c|c|c|c|c|c|c|}
\hline \multirow{2}{*}{ Trat. } & Folha & Galho & Lenho & Casca & Tronco & PA & $\mathrm{MO}$ & $\mathrm{Rg}$ & $\mathrm{Rf}$ & Raízes & Total \\
\hline & \multicolumn{11}{|c|}{ (kg/ha) } \\
\hline & \multicolumn{11}{|c|}{ Nitrogênio } \\
\hline $\mathrm{T}_{1}$ & 73,3 & 16,6 & 30,8 & 20,6 & 51,4 & 141,3 & 100,5 & 11,4 & 2,4 & 13,7 & 255,6 \\
\hline $\mathrm{T}_{2}$ & 65,5 & 13,6 & 19,1 & 16,0 & 35,1 & 114,2 & 118,4 & 7,7 & 3,8 & 11,5 & 244,1 \\
\hline $\mathrm{T}_{3}$ & 62,2 & 11,2 & 20,2 & 13,5 & 33,7 & 107,1 & 80,3 & 19,0 & 2,6 & 21,6 & 209,0 \\
\hline \multirow[t]{2}{*}{$\mathrm{T}_{4}$} & 70,5 & 13,8 & 15,58 & 10,1 & 25,7 & 110,0 & 130,6 & 10,7 & 2,4 & 13,0 & 253,7 \\
\hline & \multicolumn{11}{|c|}{ Fósforo } \\
\hline $\mathrm{T}_{1}$ & 3,8 & 1,4 & 2,2 & 2,0 & 4,2 & 9,4 & 3,6 & 0,7 & 0,2 & 0,9 & 13,9 \\
\hline $\mathrm{T}_{2}$ & 3,4 & 1,3 & 1,5 & 1,3 & 2,8 & 7,5 & 3,9 & 0,5 & 0,2 & 0,7 & 12,1 \\
\hline $\mathrm{T}_{3}$ & 3,8 & 1,2 & 1,3 & 2,2 & 3,5 & 8,5 & 3,0 & 1,6 & 0,3 & 1,8 & 13,4 \\
\hline \multirow[t]{2}{*}{$\mathrm{T}_{4}$} & 3,5 & 1,3 & 1,2 & 0,9 & 2,1 & 6,9 & 5,9 & 0,7 & 0,2 & 0,9 & 13,7 \\
\hline & \multicolumn{11}{|c|}{ Potássio } \\
\hline $\mathrm{T}_{1}$ & 18,2 & 14,2 & 32,5 & 25,3 & 57,8 & 90,2 & 6,4 & 4,3 & 0,5 & 4,9 & 101,4 \\
\hline $\mathrm{T}_{2}$ & 20,0 & 13,8 & 23,9 & 17,9 & 41,8 & 75,6 & 3,5 & 3,0 & 0,7 & 3,7 & 82,8 \\
\hline $\mathrm{T}_{3}$ & 17,4 & 10,3 & 20,8 & 14,9 & 35,7 & 63,4 & 4,9 & 9,2 & 0,6 & 9,9 & 78,1 \\
\hline \multirow[t]{2}{*}{$\mathrm{T}_{4}$} & 15,1 & 10,5 & 21,1 & 11,2 & 32,3 & 57,9 & 10,4 & 3,2 & 0,3 & 3,5 & 71,8 \\
\hline & \multicolumn{11}{|c|}{ Cálcio } \\
\hline $\mathrm{T}_{1}$ & 20,2 & 15,7 & 20,3 & 49,0 & 69,3 & 105,2 & 76,0 & 8,0 & 0,7 & 8,7 & 189,9 \\
\hline $\mathrm{T}_{2}$ & 14,0 & 10,1 & 13,5 & 19,0 & 32,5 & 56,6 & 52,1 & 3,5 & 0,7 & 4,3 & 112,9 \\
\hline $\mathrm{T}_{3}$ & 18,2 & 11,1 & 13,1 & 24,2 & 37,3 & 66,6 & 53,0 & 14,0 & 0,8 & 14,8 & 134,4 \\
\hline \multirow[t]{2}{*}{$\mathrm{T}_{4}$} & 23,0 & 16,3 & 11,9 & 38,3 & 50,2 & 89,5 & 83,0 & 8,9 & 0,9 & 9,8 & 182,3 \\
\hline & \multicolumn{11}{|c|}{ Magnésio } \\
\hline $\mathrm{T}_{1}$ & 6,8 & 3,9 & 5,6 & 8,4 & 14,0 & 24,7 & 13,7 & 2,2 & 0,4 & 2,6 & 41,0 \\
\hline $\mathrm{T}_{2}$ & 6,0 & 2,6 & 4,0 & 5,3 & 9,3 & 17,9 & 9,1 & 0,8 & 0,3 & 1,1 & 28,1 \\
\hline $\mathrm{T}_{3}$ & 9,2 & 4,7 & 5,6 & 9,8 & 15,4 & 29,3 & 19,5 & 9,4 & 0,5 & 9,9 & 58,7 \\
\hline $\mathrm{T}_{4}$ & 6,7 & 3,2 & 2,9 & 5,6 & 8,5 & 18,4 & 14,3 & 1,8 & 0,3 & 2,1 & 34,8 \\
\hline
\end{tabular}

$\mathrm{PA}=$ parte aérea $($ folha + galho + lenho + casca), $\mathrm{MO}=$ manta orgânica, $\mathrm{Rg}=$ raízes grossas $($ diâmetro $>3,00 \mathrm{~mm})$, Rf = raízes finas (diâmetro $\leq 3,00 \mathrm{~mm}$ ) e Raízes = soma de raízes grossas e raízes finas.

Os conteúdos dos nutrientes não variaram de maneira consistente com o crescimento das árvores (Quadro 6). A relação entre a biomassa (kg/ha) e o seu conteúdo de nutrientes $(\mathrm{kg} / \mathrm{ha})$, expresso em termos de eficiência de utilização, indica que no preparo mais e menos intensivo foi respectivamente de: 319 e 198 para o N; 5.868 e 3.677 para o P; 804 e 701 para o K, 429 e 276 para o Ca; e 1.889 e 1.447 (kg/kg) para o Mg, mostrando que os nutrientes foram mais eficientemente utilizados para o crescimento quando o cultivo foi mais intensivo, o que representaria sua menor exportação relativa. Por outro lado, os resíduos deixados na área do cultivo mínimo seriam mais ricos em nutrientes, o que é desejável do ponto de vista da sustentabilidade da produção florestal.

A manta orgânica foi o compartimento do sistema que acumulou maiores quantidades de todos os nutrientes, exceto K, que se acumulou mais no lenho (Quadro 6). Ela é o principal reservatório de nutrientes, em razão de seus teores relativamente elevados de nutrientes e por ser, após o lenho, o segundo componente do sistema em participação na biomassa total. Assim, o conteúdo,

R. Árvore, Viçosa-MG, v.27, n.5, p.635-646, 2003 
considerando os tratamentos aplicados, segue, em geral, a mesma tendência da biomassa, com maior quantidade de nutrientes na manta correspondente ao cultivo mínimo. Nos preparos mais e menos intensivos a manta representa 16,8 e $29,3 \%$ da biomassa total, respectivamente (Quadro 4). Quanto aos nutrientes, ela contém cerca de 44,7, 30,9, 7,5, 42,6 e 34,8\% do total de N, P, K, Ca e $\mathrm{Mg}$ presentes no sistema, respectivamente. Portanto, conclui-se que a manta orgânica representa o principal componente de plantações comerciais de eucalipto na manutenção de nutrientes na área e que as folhas, por sua intensa atividade metabólica, seriam o segundo componente na conservação de $\mathrm{N}$ e $\mathrm{P}$, apesar da pequena contribuição da biomassa total.

Considerando os vários compartimentos do sistema, as maiores quantidades de $\mathrm{P}, \mathrm{K}$ e Ca estão imobilizadas na biomassa nos tratamentos $\mathrm{T}_{1}, \mathrm{~T}_{2} \mathrm{e} \mathrm{T}_{3}$, enquanto no $\mathrm{T}_{4}$ tal fato ocorre no compartimento solo + manta orgânica, exceto para $\mathrm{Ca}$ (Quadro 7). Entre os nutrientes, o $\mathrm{Mg}$ foi o único cujo balanço foi positivo para todos os sistemas de preparo do solo. Portanto, à medida que se intensifica o preparo do solo maior preocupação deve existir quanto ao sistema de colheita. Proporcionalmente, maior exportação de nutrientes ocorrerá nos preparos mais intensivos do que nos menos intensivos à medida que o índice de colheita aumenta, o que implica maior necessidade de reposição de nutrientes ao ecossistema, via fertilização, por exemplo, para manutenção da produção florestal.

A quantidade de nutrientes exportados nos tratamentos de preparo mais intensivo do solo é maior que no menos intensivo (Quadro 8). A permanência da copa e,
Quadro 8 - Conteúdo de nutrientes (solo, raízes, mata orgânica e copa), lenho, tronco e projeção do balanço de nutrientes no sistema após a colheita

Table 8 - Amount of nutrients in the soil, roots, forest floor, crown, stemwood, trunk, as well as nutrient balance according to harvest intensity

\begin{tabular}{|c|c|c|c|c|c|}
\hline \multirow{3}{*}{ Trat. } & \multicolumn{5}{|c|}{ Conteúdo de Nutrientes nos Compartimentos } \\
\hline & $\begin{array}{c}\text { Solo + Re- } \\
\text { síduos* }\end{array}$ & Lenho & Tronco & $\begin{array}{c}\text { Solo + } \\
\text { Resíduos - } \\
\text { Lenho }\end{array}$ & $\begin{array}{c}\text { Solo + } \\
\text { Resíduos - } \\
\text { Tronco }\end{array}$ \\
\hline & \multicolumn{5}{|c|}{$(\mathrm{kg} / \mathrm{ha})$} \\
\hline & \multicolumn{5}{|c|}{ Fósforo } \\
\hline $\mathrm{T}_{1}$ & 12,1 & 2,2 & 4,2 & 9,9 & 7,9 \\
\hline $\mathrm{T}_{2}$ & 11,7 & 1,5 & 2,8 & 10,2 & 8,9 \\
\hline $\mathrm{T}_{3}$ & 12,3 & 1,3 & 3,5 & 11,0 & 8,8 \\
\hline \multirow[t]{2}{*}{$\mathrm{T}_{4}$} & 14,1 & 1,2 & 2,1 & 12,9 & 12,0 \\
\hline & \multicolumn{5}{|c|}{ Potássio } \\
\hline $\mathrm{T}_{1}$ & 106,5 & 32,5 & 57,8 & 74,0 & 48,7 \\
\hline $\mathrm{T}_{2}$ & 100,3 & $\begin{array}{ll}23,9 \\
23,9\end{array}$ & 41,8 & 76,4 & 58,5 \\
\hline $\mathrm{T}_{3}$ & 96,5 & 20,8 & 35,7 & 75,7 & 60,8 \\
\hline \multirow[t]{2}{*}{$\mathrm{T}_{4}$} & 107,0 & 21,1 & 32,3 & 85,9 & 74,7 \\
\hline & \multicolumn{5}{|c|}{ Cálcio } \\
\hline $\begin{array}{l}\mathrm{T}_{1} \\
\mathrm{~N}\end{array}$ & 134,7 & 20,3 & 69,3 & 114,4 & 65,4 \\
\hline $\mathrm{T}_{2}$ & 84,7 & 13,5 & 32,5 & 71,2 & 52,2 \\
\hline $\mathrm{T}_{3}$ & 112,8 & 13,1 & 37,3 & 99,7 & 75,5 \\
\hline \multirow[t]{2}{*}{$\mathrm{T}_{4}$} & 160,7 & 11,9 & 50,2 & 148,8 & 110,5 \\
\hline & \multicolumn{5}{|c|}{ Magnésio } \\
\hline $\begin{array}{l}\mathrm{T}_{1} \\
n_{1}\end{array}$ & 52,9 & 5,6 & 14,0 & 47,3 & 38,9 \\
\hline $\mathrm{T}_{2}$ & 38,1 & 4,0 & 9,3 & 34,1 & 28,8 \\
\hline$T_{3}$ & 68,6 & 5,6 & 15,4 & 63,0 & 53,2 \\
\hline $\mathrm{T}_{4}$ & 55,6 & 2,9 & 8,5 & 52,7 & 47,1 \\
\hline
\end{tabular}

* Solo+Resíduos $=$ solo $(0-40 \mathrm{~cm}$ prof. $)+$ raízes + manta orgânica + copa (folhas + galhos), Lenho $=$ madeira sem casca e Tronco $=$ madeira com casca .

Quadro 7 - Quantidade e distribuição relativa de P, K, Ca e Mg no sistema solo, manta orgânica e planta em povoamentos de Eucalyptus grandis, aos 38 meses de idade, influenciadas pelo preparo do solo adotado para reforma de floresta

Table 7 - Amount and relative distribution of $P, K, C a$ and $M g$ in the soil, forest floor and 38 month old trees of Eucalyptus grandis, as affected by soil tillage systems

\begin{tabular}{|c|c|c|c|c|c|c|c|c|c|c|c|c|c|c|c|c|}
\hline \multirow{3}{*}{ Trat. } & \multicolumn{4}{|c|}{ Prof. do Solo $(0-40 \mathrm{~cm})$} & \multicolumn{4}{|c|}{ Manta Orgânica } & \multicolumn{4}{|c|}{ Biomassa } & \multicolumn{4}{|c|}{ Total no Sistema } \\
\hline & $\mathrm{P}$ & $\mathrm{K}$ & $\mathrm{Ca}$ & $\mathrm{Mg}$ & $\mathrm{P}$ & $\mathrm{K}$ & $\mathrm{Ca}$ & $\mathrm{Mg}$ & $P$ & $\mathrm{~K}$ & $\mathrm{Ca}$ & $\mathrm{Mg}$ & $\mathrm{P}$ & $\mathrm{K}$ & $\mathrm{Ca}$ & $\mathrm{Mg}$ \\
\hline & \multicolumn{16}{|c|}{$(\mathrm{kg} / \mathrm{ha})$} \\
\hline \multirow{2}{*}{$\mathrm{T}_{1}$} & 2,4 & 62,8 & 14,1 & 25,9 & 3,6 & 6,4 & 76,0 & 13,7 & 10,3 & 94,9 & 113,9 & 27,3 & 16,3 & 164,1 & 204,0 & 66,9 \\
\hline & $(15,0)$ & $(38,3)$ & $(6,9)$ & $(38,7)$ & $(22,0)$ & $(3,9)$ & $(37,3)$ & $(20,5)$ & $(63,0)$ & $(57,9)$ & $(55,9)$ & $(40,7)$ & $(100)$ & $(100)$ & $(100)$ & $(100)$ \\
\hline \multirow{2}{*}{$\mathrm{T}_{2}$} & 2,4 & 59,3 & 4,2 & 19,3 & 3,9 & 3,5 & 52,1 & 9,1 & 8,2 & 79,3 & 60,9 & 19,0 & 14,5 & 142,1 & 117,2 & 47,4 \\
\hline & $(16,8)$ & $(41,7)$ & $(3,5)$ & $(40,7)$ & $(26,6)$ & $(2,5)$ & $(44,5)$ & $(19,2)$ & $(56,6)$ & $(55,8)$ & $(51,9)$ & $(40,1)$ & $(100)$ & $(100)$ & $(100)$ & $(100)$ \\
\hline \multirow{2}{*}{$\mathrm{T}_{3}$} & 2,5 & 54,0 & 15,7 & 25,3 & 3,0 & 4,9 & 53,0 & 19,5 & 10,4 & 73,3 & 81,4 & 39,1 & 15,9 & 132,2 & 150,1 & 83,9 \\
\hline & $(15,7)$ & $(40,9)$ & $(10,4)$ & $(30,0)$ & $(19,0)$ & $(3,7)$ & $(35,3)$ & $(23,3)$ & $(65,4)$ & $(55,5)$ & $(54,2)$ & $(46,6)$ & (100) & $(100)$ & (100) & $(100)$ \\
\hline \multirow{2}{*}{$\mathrm{T}_{4}$} & 2,5 & 67,5 & 28,6 & 29,3 & 5,9 & 10,4 & 83,0 & 14,3 & 7,8 & 61,4 & 99,3 & 20,6 & 16,2 & 139,3 & 210,9 & 64,2 \\
\hline & $(15,3)$ & $(48,5)$ & $(13,5)$ & $(45,6)$ & $(36,4)$ & $(7,5)$ & $(39,4)$ & $(22,3)$ & $(48,3)$ & $(44,1)$ & $(47,1)$ & $(32,1)$ & (100) & $(100)$ & (100) & $(100)$ \\
\hline
\end{tabular}

Valores entre parêntesis $=$ distribuição relativa de nutrientes no sistema. 
em especial, da casca no campo com a colheita somente do lenho permite maior sustentabilidade do sistema, em virtude da manutenção de nutrientes na área. Os resíduos atuam como reserva de nutrientes, pois podem ainda, mesmo que em parte, atender à demanda das plantas no próximo ciclo de plantio. Esse fato foi também constatado para a mesma espécie e região, por Leite (1996) e Amaral (1999).

Entre os tratamentos avaliados, após a colheita do lenho ou do tronco o cultivo mínimo foi o que apresentou maior estoque de nutrientes, com exceção de $\mathrm{Mg}$, no sistema solo + resíduos (Quadro 8). Portanto, espera-se que, a longo prazo, o crescimento da floresta seja equivalente ou mesmo maior que o da atual, graças ao maior estoque de nutrientes no sistema.

\section{CONCLUSÃO}

Os métodos de preparo do solo utilizados para reforma de floresta de Eucalyptus grandis interferiram nas características físicas e químicas do solo, com reflexo nas características dendrométricas e na produção de biomassa.

O preparo mais intensivo do solo contribuiu para o maior crescimento das árvores, pela maior disponibilização de nutrientes e pela redução das plantas competidoras.

A alocação de carbono orgânico para produção de raízes finas tendeu a ser maior nos tratamentos com maior intensidade de manejo.

O cultivo mínimo foi o único tratamento que apresentou balanço positivo para todos os nutrientes analisados, com exceção do $\mathrm{Mg}$.

\section{REFERÊNCIAS BIBLIOGRÁFICAS}

AMARAL, G. Influência de características químicas e físicas de cinco diferentes solos na zona metalúrgica mineira na produtividade de eucalipto. 1999. $109 \mathrm{f}$. Dissertação (Mestrado em Solos e Nutrição de Plantas) Universidade Federal de Viçosa, Viçosa, 1999.

ARDENGHI, A. F. Efeito de métodos de preparo do solo e de controle de plantas daninhas sobre propriedades físicas e químicas de um Latossolo Roxo Eutrófico, cultivado com milho, durante dez anos. 1989. $68 \mathrm{f}$. Dissertação (Mestrado em Solos e Nutrição de Plantas) Universidade Federal de Viçosa, Viçosa, 1989.
BARROS, N. F.; NOVAIS, R. F.; NEVES, J. C. L. Fertilização e correção do solo para o plantio de eucalipto. In: BARROS, N. F.; NOVAIS, R. F. (Eds.). Relação soloeucalipto. Viçosa, MG: Folha de Viçosa, 1990. p. 127-186.

BINKLEY, D. Forest nutrition management. New York: John Wiley \& Sons, 1986. 290 p.

BÖHM, W. Methods of studying root systems. Berlin: Springer, 1979. $188 \mathrm{p}$.

BRAGA, J. M.; DEFELIPO, B. V. Determinação espectrofométrica de fósforo em extratos de solo e plantas. Revista Ceres, v. 21, n. 1, p. 73-85, 1974.

COSTA, L. M. Manejo de solos em áreas reflorestadas. In: BARROS, N. F.; NOVAIS, R. F. (Eds.). Relação soloeucalipto. Viçosa, MG: Folha de Viçosa, 1990. p. 237-264.

DRUMOMD, M. A. Alterações fitossociológicas e edáficas decorrentes de modificações da cobertura vegetal na Mata Atlântica, Região do Médio Rio Doce, MG. 1996. 73 f. Tese (Doutorado em Ciência Florestal) Universidade Federal de Viçosa, Viçosa, 1996.

EMPRESA BRASILEIRA DE PESQUISA AGROPECUÁRIA - EMBRAPA. Manual de métodos de análise de solo. 2.ed. Rio de Janeiro: 1997. 212 p.

GOLLEY, F. B. et al. Ciclagem de minerais em um ecossistema de floresta tropical úmida. São Paulo: EPU/ EDUSP, 1978. $256 \mathrm{p}$.

GONÇALVES, J. L. M. Efeito do cultivo mínimo sobre a fertilidade do solo e ciclagem de nutrientes. In: SEMINÁRIO SOBRE CULTIVO MÍNIMO DO SOLO EM FLORESTAS, 1., 1995, Curitiba. Anais... Piracicaba: CNPFloresta, IPEF, UNESP, SIF, FUPEF, 1995. p. 43-62.

GONÇALVES, J. L. M. et al. Reflexos do cultivo mínimo e intensivo do solo em sua fertilidade e na nutrição das árvores. In: GONÇALVES, J. L. M., BENEDETTI, V. (Eds.). Nutrição e fertilização florestal. Piracicaba: IPEF, 2000. p. 3-57.

LAZARI, M. F. Nitrificação em solos sob plantações de eucalipto com diferentes idades. 2001. 42 p. Dissertação (Mestrado em Microbiologia Agrícola) - Universidade Federal de Viçosa, Viçosa, 2001.

LEITE, F. P. Crescimento, relações hídricas, nutricionais e lumínicas em povoamento de Eucalyptus grandis em diferentes densidades populacionais. 1996. $90 \mathrm{f}$.

Dissertação (Mestrado em Solos e Nutrição de Plantas) Universidade Federal de Viçosa, Viçosa, 1996.

R. Árvore, Viçosa-MG, v.27, n.5, p.635-646, 2003 
LEITE, F. P. Relações nutricionais e alterações de características químicas de solos da Região do Vale do Rio Doce pelo cultivo do eucalipto. 2001. $72 \mathrm{f}$. Tese (Doutorado em Solos e Nutrição de Plantas) - Universidade Federal de Viçosa, Viçosa, 2001.

MALUF, L. L. P. Efeito da queima, métodos de preparo do solo e da adubação no crescimento de $E$. camaldulensis em Areia Quartzosa. 1991. 78 f. Dissertação (Mestrado em Solos e Nutrição de Plantas) - Universidade Federal de Viçosa, Viçosa, 1991.

NEVES, J. C. L. Produção e partição de biomassa, aspectos nutricionais e hídricos em plantios locais de eucalipto na região litorânea do Espírito Santo. 2001. 192 f. Tese (Doutorado) - Universidade Estadual Norte Fluminense, Campos dos Goitacases 2001.

NOVAIS, R. F.; BARROS, N. F.; NEVES, J. C. L. Interpretação de análise química do solo para o crescimento de Eucalyptus spp. - Níveis críticos de implantação e de manutenção. Revista Árvore, v. 10, n. 1, p. 105-111, 1986.
REIS, G. G.; REIS, M. G. F. Reflexos do cultivo mínimo no ambiente e na fisiologia da árvore. In: SEMINÁRIO SOBRE CULTIVO MÍNIMO DO SOLO EM FLORESTAS, 1., 1995, Curitiba. Anais... Curitiba: CNPFloresta/IPEF/ UNESP/SIF/FUPEF, 1995. p. 148-162.

SANTANA, R. C.; NEVES, J. C. L. Produção de biomassa e conteúdo de nutrientes de procedência de Eucalyptus grandis e Eucalyptus saligna em alguns sítios do estado de São Paulo. Scientia Forestalis, n. 56, p. 155-169, 1999.

SCHENEIDER, P. R. Introdução ao manejo florestal. Santa Maria: UFSM, 1993. 348 p.

TENNANT, D. A. Test of a modified line intersect method of estimating root length. Journal of Ecology, v. 63, n. 3, p. $995-1001,1975$.

TOMÉ JUNIOR, J. B. Manual para interpretação de análise de solo. Guaíba: Agropecuária, 1997. 247 p. 